\title{
Estimated additional lifetime risk of cancer attributable to diagnostic $C T$ in a pediatric bone marrow transplant cohort: Experience at a single academic institution
}

\author{
Jonathan O. Swanson ${ }^{1 *}$, Adam Alessio ${ }^{2}$, Teresa Chapman ${ }^{1}$ \\ ${ }^{1}$ Department of Radiology, Seattle Children’s Hospital/University of Washington, Seattle, USA \\ ${ }^{2}$ Department of Radiology, University of Washington, Seattle, USA \\ Email: *jonathan.swanson@seattlechildrens.org
}

Received 5 December 2011; revised 21 December 2011; accepted 2 February 2012

\begin{abstract}
Purpose: To determine the frequency of CT procedures in a cohort of bone marrow transplant patients and estimate the effective dose from each CT procedure as well as rough estimates of lifetime attributable risk (LAR) of cancer (both incidence and mortality). Background: Pediatric patients who undergo bone marrow transplant benefit greatly from the diagnostic power of computed tomography, but due to the need for frequent imaging, these patients are repeatedly exposed to the carcinogenic potential of ionizing radiation. Methods: CT Imaging and patient parameters were collected from a retrospective cohort of bone marrow transplant patients. Dosimetry was estimated as a function of age, dose length product (DLP), and scan region based on published DLP to effective dose tables. Lifetime attributable risk (LAR) of cancer as a function of age, gender, and organ specific dose was derived from BEIR VII phase 2 estimates. Results: 44 patients with bone marrow transplant were included and ranged in age from 7 months to 20 years (average age, 9 years). The average number of CT studies per patient was 3.2 over the 15 month period. The average effective dose for each study was $5.9+/-4.5 \mathrm{mSv}$. Cumulative effective dose to each patient was $20+/-32 \mathrm{mSv}$. It was estimated that in this cohort, the CT imaging performed over a 15-month period on a 64-slice scanner led to a lifetime additional risk of cancer incidence of 5 in 1000 and a lifetime additional risk of cancer mortality of 2 in 1000. Conclusion: Diagnostic CT is important in the assessment and management of ill patients following bone marrow transplant. The risk of ionizing radiation leading to additional development of cancer merits using as low a CT technique as reasonable to achieve a diagnostic study.
\end{abstract}

\footnotetext{
${ }^{*}$ Corresponding author.
}

Keywords: Ionizing Radiation; Effective Dose; CT; Lifetime Additional Risk

\section{INTRODUCTION}

The evolution of computed tomography technology in the last 20 years has markedly improved our ability to image children. CT innovations have decreased scanning duration and improved spatial and temporal resolution [1]. These innovations, in turn, have reduced the need for sedation and have allowed the imaging of younger, sicker and less cooperative children [1,2]. Benefits of CT imaging, including avoidance of unnecessary exploratory laparotomies, the ability to diagnose disease earlier in its course, and the tracking of disease progression, have helped drive the rapid increase of CT use in children.

The commonly acknowledged primary risk of computed tomography is the increased potential for subsequent development of cancer [3-8]. Exposure to lowdose ionizing radiation has been shown to increase the incidence of cancer in the life long-studied cohort of Hiroshima and Nagasaki atomic bomb survivors, as well as in both occupational exposure studies and in medical radiation therapy studies. The pediatric population is at a greater risk for development of subsequent cancer following ionizing radiation exposure than are adults due to the presence of more radiosensitive developing tissues and to the longer period of time that a radiation-induced cancer has to manifest. It should be stressed that the current knowledge on the risks associated with whole-body radiation doses below $100 \mathrm{mSv}$ is extremely limited. The estimates presented here are based on this limited knowledge and theory and should not be construed as exact estimates. With this in mind, this effort intends to demonstrate that even with conservative estimates, the risk of cancer is extremely low and still warrants even lower CT techniques

Within the pediatric population, patients at greatest 
risk are those who undergo CT procedures with the greatest frequency [5]. Chronic illnesses are being successfully managed with advancing medicine [9,10], but may be accompanied by complications and the need for routine surveillance that require diagnostic imaging. Bone marrow transplant (BMT) patients are an excellent example of such a pediatric patient population. The post transplant period in BMT patients is complicated by infection, graft versus host disease, and secondary malignnancies, requiring a high frequency of diagnostic CT imaging for rapid, accurate diagnoses.

To the best of our knowledge, there is no study that investigates the cumulative radiation exposure of diagnostic CT in this particular population. This study addresses radiation exposure in BMT patients and provides an estimate of the additional lifetime risk of cancer attributable to diagnostic CT in a small cohort of pediatric patients who have undergone bone marrow transplantation.

Here we aim to determine the frequency of CT procedures in a cohort of bone marrow transplant patients treated here at Seattle Children's Hospital. We estimate the effective dose from each CT procedure, and then extrapolate and estimate the lifetime attributable risk (LAR) of cancer (both incidence and mortality).

\section{METHODS}

IRB approval was obtained for this retrospective analysis. A search for pediatric patients who had undergone bone marrow transplantation and who were subsequently imaged by CT between August 1, 2007 to October 31, 2008 was performed using a radiology information system. CT imaging parameters were collected for all diagnostic CT studies performed for each patient on a 64-slice multidetector CT (GE Lightspeed VCT; GE Healthcare, Waukesha, WI).

Organ specific dosimetry was estimated as a function of age, dose length product (DLP), tube voltage $(\mathrm{kVp})$, and scan region based on a published method [11]. This method is derived from accepted DLP to dose factors originally derived from Monte Carlo simulations [12] and employed in numerous publications [13-15]. In brief, we determined the DLP $(\mathrm{mGy} \times \mathrm{cm})$ to organ specific dose (mGy) conversion factors for six common CT scan regions with the ImPACT CT Dosimetry Calculator (version 1.0.2, ImPACT, London, England), which draws estimates from Monte Carlo simulations of reference adult patient [16]. These conversion factors were modified for pediatric models based on proposed relationships [17]. Furthermore, the factors were adjusted slightly to account for the absorbed dose dependency on CT tube voltage as proposed by Huda [18]. After estimation of organ specific doses, the effective dose was derived from the cumulative sum of organ specific doses times organ weighting factors from the International Commission on Radiological Protection (ICRP) publication 103 [19].

Lifetime attributable risk (LAR) of cancer as a function of age and gender were derived from BEIR VII phase 2 estimates. The organ-specific radiation doses where converted to organ-specific cancer risks and summed to estimate total cancer risk. This method is discussed in detail by Alessio and Phillips [11]. In our work, the organ specific risk factors were generated by linearly interpolating between age categories the gender- and organ-specific risk estimates as tabulated in the BEIR VII phase 2 tables 12D-1 for incidence and 12D-2 for mortality. The cumulative lifetime attributable risk (LAR) is the sum of risk for all forms of cancer. These risk estimates are based on the current, limited knowledge of risk associated with low-levels of ionizing radiation which is predicated on the linear no-threshold model and have lead to numerous CT derived cancer risk estimates in the literature $[5,13]$.

\section{RESULTS}

\subsection{Patient Cohort}

44 patients with bone marrow transplant were included and ranged in age from 7 months to 20 years (average age, 9 years). Indications for bone marrow transplant included acute leukemia $(\mathrm{n}=19)$, chronic leukemia $(\mathrm{n}=$ $2)$, lymphoma $(\mathrm{n}=1)$, immunodeficiency syndrome $(\mathrm{n}=$ 14), myelodysplastic syndrome $(n=2)$, high-risk solid tumor $(\mathrm{n}=1)$, hemophagocyticlymphohistiocytosis ( $\mathrm{n}=$ $3)$, and osteopetrosis $(n=2)$.

\subsection{Diagnostic CT}

Indications for diagnostic CT in our patient population are listed in Table 1. The number of CT studies performed on any single patient within this time frame was 1 - 13 procedures, and the average number of CT studies was 3.2 per 15 months. The average Effective Dose (ED) per study was $5.9+/-4.5 \mathrm{mSv}$. The cumulative ED to each patient was on average $20+/-32 \mathrm{mSv}$ with a median of $11 \mathrm{mSv}$.

The CT protocols are defined based on 11 weightbased categories [20]. In each weight category, the average age based on standard growth charts and the average DLP for chest studies in this category was determined to estimate the effective dose from a chest CT in for each protocol (Table 2). These effective dose estimates are plotted in Figure 1.

\section{DISCUSSION}

In this study, the frequency of diagnostic CT procedures in a cohort of bone marrow transplant patients between 
Table 1. Breakdown of the varied indications for diagnostic CT in our patient population.

\begin{tabular}{lc}
\hline \multicolumn{1}{c}{ Indications for CT } & Frequency \\
\hline Fever & $\mathbf{3 6 . 4 \%}$ \\
Follow-up Pulmonary Opacity & $\mathbf{1 4 . 6 \%}$ \\
Respiratory Distress & $\mathbf{1 1 . 9 \%}$ \\
Follow-up Pulmonary Nodule & $\mathbf{8 . 0 \%}$ \\
Abdominal Pain & $\mathbf{6 . 7 \%}$ \\
Follow-up GVHD & $\mathbf{4 . 0 \%}$ \\
Concern for EBV/PTLD & \\
Intracranial Hemorrhage & $\mathbf{2 . 3 \%}$ \\
Headache & $\mathbf{2 . 3 \%}$ \\
Sinus Disease Follow-up & $\mathbf{1 . 3 \%}$ \\
Other & $\mathbf{1 . 3 \%}$ \\
\hline
\end{tabular}

*GVHD—Graft versus host disease; ${ }^{* *}$ PTLD—Post transplant lymphoproliferative disease.

In this study, the frequency of diagnostic CT procedures in a cohort of bone marrow transplant patients between the ages of 7 months and 20 years was retrospectively observed, and the average effective dose per patient was calculated. Using data from the BEIR VII study, a gross approximation of the additional risk of cancer attributable to these diagnostic CT studies was calculated. This particular patient group is of interest because of the unusually high rate of complications that may be seen following bone marrow transplantation, requiring diagnostic imaging.
The average total effective dose per diagnostic CT procedurein our study, which included both head and body imaging, was $5.9+/-4.5 \mathrm{mSv}$. It was estimated that in this cohort, the CT imaging performed over a 15month period on a 64-slice scanner led to on average an additional lifetime additional risk of cancer incidence of 34 in 10,000 and a lifetime additional risk of cancer mortality of 17 in 10,000. For perspective, in the absence of ionizing radiation, the background overall risk of a cancer death in the USA over a person's lifetime is $20 \%$ $25 \%$ ( 2500 in 10,000). Estimates for LAR for cancer incidence for females for a single chest CT acquisition versus patient age are plotted in Figure 2.

Comparable assessments of dose and risk have been performed on other subsets of the pediatric population. Huang et al. [21]evaluated dose related to multi-detector CT for coronary angiography and reported much higher effective doses in the 5-year-old child compared to what we observed in our patient population. This relatively high dose is expected given the higher dose required for detailing coronary arterial anatomy. The EDs ranged from 11.81 - $16.45 \mathrm{mSv}$, depending on heart rate. This group also estimated the LAR from coronary MDCT, which was much higher in girls than in boys $(0.43 \%$ $0.60 \%$ in girls; $0.14 \%-0.20 \%$ in boys). Feng et al. [1] used phantom data to show that the doses from common pediatric CT examinations using a 64-slice MDCT ranged from $0.7 \mathrm{mSv}$ to $3.5 \mathrm{mSv}$ and the associated lifetime cancer risks were found to be up to $0.16 \%$.These values were similar to the $0.06 \%$ estimation of lifetime attribut-

Table 2. Summary of diagnostic chest protocols performed during this study. Protocols are presented for 11 patient weight categories (All protocols used proper bow-tie filters are selected to reduce skin dose. All pitches are at 1.375 and obtained at $64 \times 0.625 \mathrm{~mm}$ ). All dosimetry is approximate based on interpolation of age specific models and acquisition settings. Cancer risk is based on estimates from organ-specific doses. For reference, according to the BEIR VII report, the baseline lifetime attributable risk of cancer incidence is approximately $45.5 \%$ for men and $36.9 \%$ for women. The presented factors are estimates of additional LAR.

\begin{tabular}{|c|c|c|c|c|c|c|c|c|}
\hline \multicolumn{3}{|c|}{ Patient } & \multicolumn{2}{|c|}{ CT Technique } & \multicolumn{2}{|c|}{ Approximate Dosimetry ${ }^{a}$} & \multicolumn{2}{|c|}{$\begin{array}{l}\text { Additional LAR of cancer inci- } \\
\text { dence attributable to exposure (\%) }\end{array}$} \\
\hline COLOR & Kilograms & $\begin{array}{l}\text { Average Age } \\
\text { (years) }\end{array}$ & $\mathbf{k V p}$ & $\begin{array}{c}\text { mA range @ } \\
0.5 \mathrm{sec}\end{array}$ & $\begin{array}{l}\text { Effective CT Dose } \\
\quad(\mathrm{mSv})[1,2]\end{array}$ & $\begin{array}{l}\text { Background Equivalent } \\
\text { Radiation Time (years) }\end{array}$ & Male & Female \\
\hline PINK & $6-7.4 \mathrm{~kg}$ & 4 months & 100 & $50-70$ & 4.0 & 1.3 & 0.06 & 0.21 \\
\hline RED & $7.5-9.4 \mathrm{~kg}$ & 8 months & 100 & $50-90$ & 4.6 & 1.5 & 0.06 & 0.24 \\
\hline PURPLE & $9.5-11.4 \mathrm{~kg}$ & 15 months & 100 & $50-100$ & 4.3 & 1.4 & 0.06 & 0.22 \\
\hline YELLOW & $11.5-14.4 \mathrm{~kg}$ & 2.4 & 100 & $50-100$ & 3.9 & 1.3 & 0.05 & 0.19 \\
\hline WHITE & $14.5-18.4 \mathrm{~kg}$ & 3.8 & 100 & $50-110$ & 4.0 & 1.3 & 0.05 & 0.18 \\
\hline BLUE & $18.5-22.4 \mathrm{~kg}$ & 5.9 & 100 & $50-110$ & 3.3 & 1.1 & 0.03 & 0.14 \\
\hline ORANGE & $22.5-31.4 \mathrm{~kg}$ & 8.5 & 120 & $70-120$ & 4.6 & 1.5 & 0.04 & 0.17 \\
\hline GREEN & $31.5-40.5 \mathrm{~kg}$ & 10.1 & 120 & $70-150$ & 5.0 & 1.7 & 0.04 & 0.16 \\
\hline BLACK & $40.5-55 \mathrm{~kg}$ & 12.8 & 120 & $70-240$ & 7.2 & 2.4 & 0.06 & 0.22 \\
\hline Sm Adult & $55-70 \mathrm{~kg}$ & 15.0 & 120 & $80-300$ & 7.6 & 2.5 & 0.05 & 0.20 \\
\hline Lg Adult & $>70 \mathrm{~kg}$ & 20.0 & 120 & $80-300$ & 5.3 & 1.8 & 0.03 & 0.11 \\
\hline
\end{tabular}




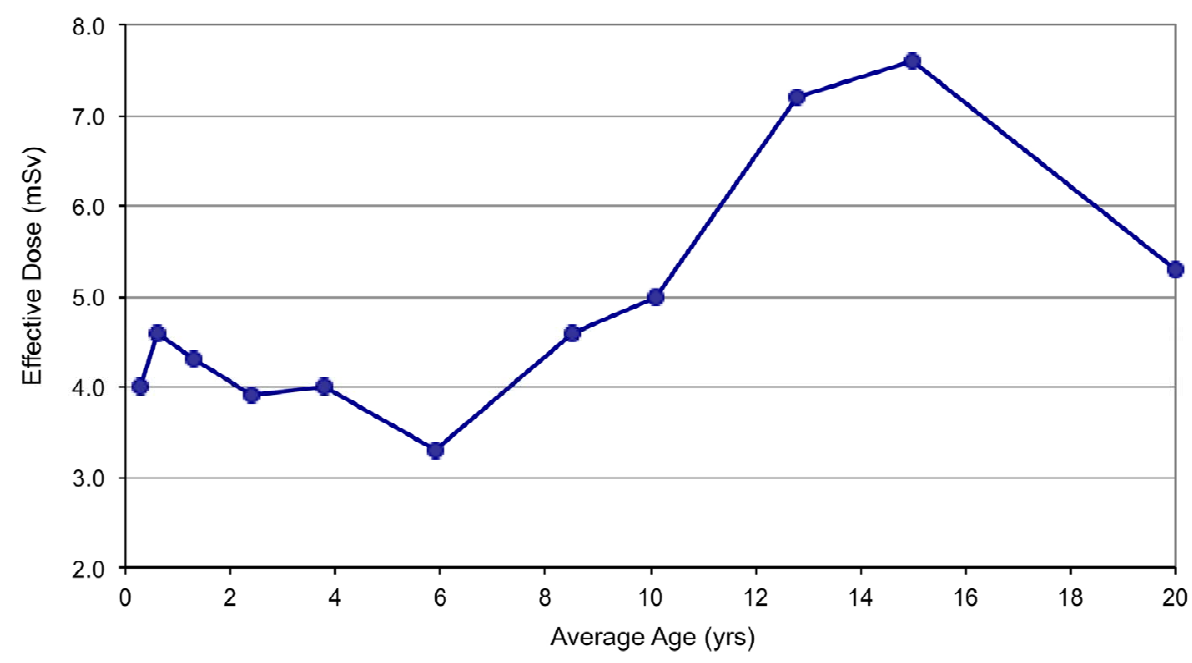

Figure 1. Estimated effective dose from a standard chest CT acquisition for each age/weight category.

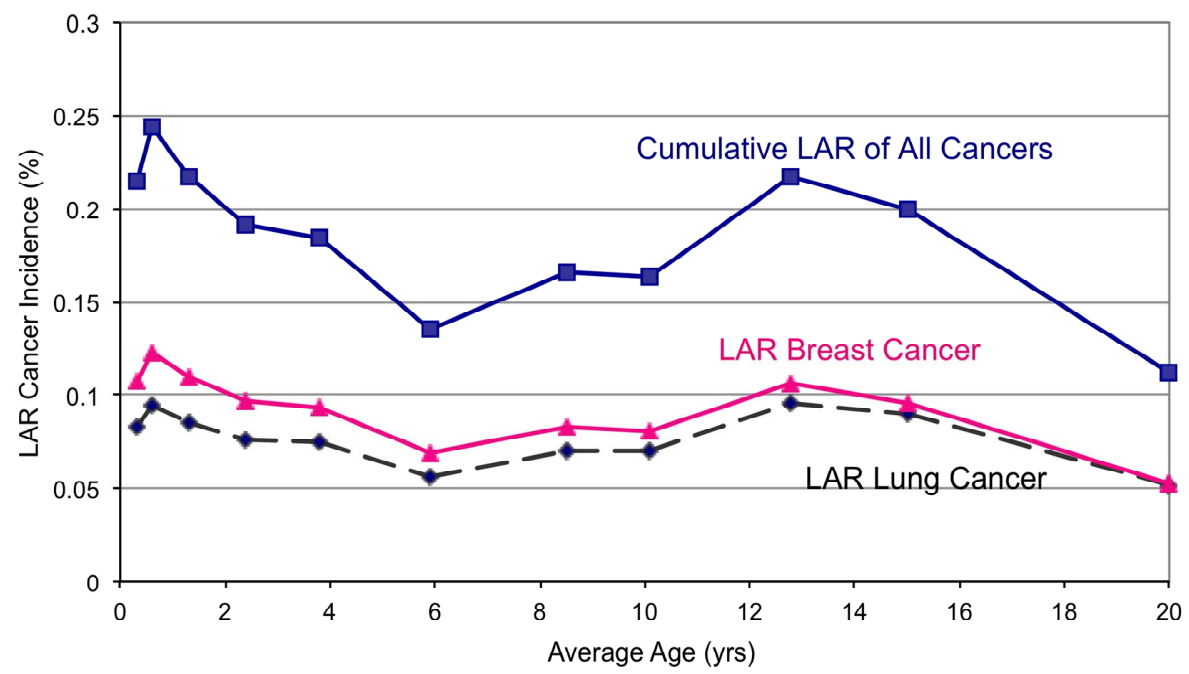

Figure 2. Estimates of additional lifetime attributable risk of cancer incidence from chest CT exam in females. These are based on extremely conservative estimates presented in BEIR VII phase 2 report and the methodology previously presented [11].

able risk of cancer incidence from low-dose chest CT for 5-year-old children [22].

More pertinent reviews have been performed on pediatric cancer populations. Pierobon et al. [23] reported the cumulative effective radiation dose associated with radiographic and radioisotopic procedures for 81 pediatric patients with malignant lymphoma over their course of diagnosis, treatment and follow-up. Follow-up periods of the survivors ranged from 2.4 to 12.3 years, and the median effective dose was $309 \mathrm{mSv}$ for non-Hodgkin lymphoma and $518 \mathrm{mSv}$ for Hodgkin lymphoma. These values are much higher than the values we publish in our patient population, even accounting for the 10 -fold longer time of study, accountable in part due to nuclear medicine studies performed. Another published review of pediatric cancer patients estimated the radiation dose attributable to PET-CT over a five-year period [24]. The average cumulative radiation dose per patient was 78.9 $\mathrm{mSv}$. Neither of these groups estimated the additional lifetime attributable risk of cancer to these diagnostic studies.

By providing an estimation of risk secondary to CT exams in pediatric BMT patients, this study can be used to refocus our attention on how to lower the radiation exposure to this patient population without decreasing our diagnostic accuracy. The most effective way to reduce dose is to reduce the number of exams. However, pediatric BMT patients are immunocompromised, and so frequent imaging of this patient population is largely justified. If the number of studies cannot be reduced, we 
could consider alternative modalities, such as ultrasound and magnetic resonance imaging, especially for head and abdominal imaging. Yet, for a large percentage of the studies for pediatric BMT patients, CT will remain the best tool. Thus, future efforts may be best focused on reducing dose per CT exam. At our institution, we are employing noise insertion software, as discussed by Karmazyn et al. [25], to simulate lower doses of chest CT and evaluate diagnostic accuracy for detection of pulmonary nodules. This work will lead to protocols with sufficient diagnostic utility at a minimum of dose. An alternative approach may be the gradual transition to using CT scanners with iterative reconstruction techniques for this patient population. Iterative reconstruction techniques have demonstrated the potential for improving image quality and reducing radiation dose in CT relative to the currently used filtered back projection techniques [26-28].

Our study is limited by the inhererent uncertainties of calculating cancer risk from low-dose radiation exposure. As detailed in Linet et al. [29], the relationship between radiation and cancer risk can be skewed by confounding factors that are associated with both exposure and disease, but not part of the causal pathway. Models of radiation exposure and cancer risk are subject to a number of statistical uncertainties, and for accurate assessment of these risks, uncertainties should be taken into account $[29,30]$.Uncertainties also arise from the method of calculating the effective doses and LAR [1,31]. As with the estimates by Feng et al., we could be underestimating the effective doses, because the radio sensitivity of pediatric organs and tissues are higher compared to adults [1]. Reference factors for specific organ sensitivities of varying ages, however, have yet to be developed for more appropriate estimation and application of effective dose in pediatric individuals $[1,19]$.

Diagnostic CT is important in the assessment and management of ill patients following bone marrow transplant. The risk of ionizing radiation leading to additional development of cancer merits using as low a CT technique as reasonable to achieve a diagnostic study. The evaluation presented here provides the imaging trends of bone marrow transplant pediatric patients at a single academic institution. As CT procedures are increasingly being evaluated for dose reduction while maintaining diagnostic specificity, such an inquiry may be of significant assistance to this population with the goal of decreasing lifetime cancer risk.

\section{REFERENCES}

[1] Feng, S.-T., Law, M.W.-M., Huang, B., Ng, S., Li, Z.-P., Meng, Q.-F. and Khong, P.-L. (2010) Radiation dose and cancer risk from pediatric CT examinations on 64-slice
CT: A phantom study. European Journal of Radiology, 76, 19-23. doi:10.1016/j.ejrad.2010.03.005

[2] Papadakis, A.E., Perisinakis, K. and Damilakis, J. (2008) Automatic exposure control in pediatric and adult multidetector CT examinations: A phantom study on dose reduction and image quality. Medical Physics, 35, 45674566. doi:10.1118/1.2977535

[3] Amis, E.S., Butler, P.F., Applegate, K.E., Birnbaum, S.B., Brateman, L.F., Hevezi, J.M., Mettler, F.A., Morin, R.L., Pentecost, M.J., Smith, G.G., et al. (2007) American College of Radiology white paper on radiation dose in medicine. Journal of the American College of Radiology, 4, 272-284. doi:10.1016/j.jacr.2007.03.002

[4] Brenner, D.J. and Elliston, C.D. (2004) Estimated radiation risks potentially associated with full-body CT screening. Radiology, 232, 735-738. doi:10.1148/radiol.2323031095

[5] Brenner, D.J. and Hall, E.J. (2007) Computed tomography-An increasing source of radiation exposure. The New England Journal of Medicine, 357, 2277-2284. doi:10.1056/NEJMra072149

[6] Goske, M.J., Applegate, K.E., Boylan, J., Butler, P.F., Callahan, M.J., Coley, B.D., Farley, S., Frush, D.P., Hernanz-Schulman, M., Jaramillo, D., et al. (2008) The image gently campaign: Working together to change practice. American Journal of Roentgenology, 190, 273-274. doi:10.2214/AJR.07.3526

[7] Linton, O.W. and Mettler, F.A. Jr. (2003) National conference on dose reduction in CT, with an emphasis on pediatric patients. American Journal of Roentgenology, 181 321-329.

[8] The National Research Council's Committees on the Biological Effects of Ionizing Radiations Committee (2006) Health risks from exposure to low levels of ionizing radiation-BEIR VII phase 2. The National Academies Press, Washington DC.

[9] Anderson, G. and Horvath, J. (2004) The growing burden of chronic disease in America. Public Health Reports, 119, 263-270.

[10] Newacheck, P.W. and Taylor, W.R. (1992) Childhood chronic illness: Prevalence, severity, and impact. American Journal of Public Health, 82, 364-371. doi:10.2105/AJPH.82.3.364

[11] Alessio, A.M. and Phillips, G.S. (2010) A pediatric CT dose and risk estimator. Pediatric Radiology, 40, 18161821. doi:10.1007/s00247-010-1761-0

[12] Shrimpton, P. (1997) Reference doses for computed tomograpny. In: Board, N.R.P., Ed., Radiological Protection Bulletin, Chilton, England, pp. 16-19.

[13] Einstein, A.J., Henzlova, M.J. and Rajagopalan, S. (2007) Estimating risk of cancer associated with radiation exposure from 64-slice computed tomography coronary angiography. The Journal of the American Medical Association, 298, 317-323. doi:10.1001/jama.298.3.317

[14] Shrimpton, P. (2009) Effective dose and dose-length product in CT. Radiology, 250, 604-605. doi:10.1148/radiol.2502081340

[15] Thomas, K.E. and Wang, B. (2008) Age-specific effect- 
tive doses for pediatric MSCT examinations at a large children's hospital using DLP conversion coefficients: A simple estimation method. Pediatric Radiology, 38, 645656. doi:10.1007/s00247-008-0794-0

[16] Shrimpton, P.C. and Jones, D.G. (1993) Normalised organ doses for x-ray computed tomography calculated using monte carlo techniques and a mathematical anthropomorphic phantom. Radiation Protection Dosimetry, 49, 241-243.

[17] Khursheed, A., Hillier, M.C., Shrimpton, P.C. and Wall, B.F. (2002) Influence of patient age on normalized effecttive doses calculated for CT examinations. British Journal of Radiology, 75, 819-830.

[18] Huda, W., Ogden, K.M. and Khorasani, M.R. (2008) Converting dose-length product to effective dose at CT. Radiology, 248, 995-1003. doi:10.1148/radiol.2483071964

[19] International Commission on Radiological Protection publication 103 (2007) The 2007 recommendations of the international commission on radiological protecttion. Annals of the ICRP, 37, 1-332.

[20] Frush, D.P., Soden, B., Frush, K.S. and Lowry, C. (2002) Improved pediatric multidetector body CT using a sizebased color-coded format. American Journal of Roentgenology, 178, 721-726.

[21] Huang, B., Law, M.W.-M., Mak, H.K.-F., Kwok, S.P.-F. and Khong, P.-L. (2009) Pediatric 64-MDCT coronary angiography with ECG-modulated tube current: Radiation dose and cancer risk. American Journal of Roentgenology, 193, 539-544. doi:10.2214/AJR.08.1920

[22] Huda, W. (2007) Radiation doses and risks in chest computed tomography examinations. Proceedings of the American Thoracic Society, 4, 316-320. doi:10.1513/pats.200611-172HT

[23] Pierobon, J., Webber, C.E., Nayiager, T., Barr, R.D., Moran, G.R. and Gulenchyn, K.Y. (2011) Radiation doses originnating from diagnostic procedures during the treatment and follow-up of children and adolescents with malignant lymphoma. Journal of Radiological Protection, 31, 83-93. doi:10.1088/0952-4746/31/1/005
[24] Chawla, S.C., Federman, N., Zhang, D., Nagata, K., Nuthakki, S., McNitt-Gray, M. and Boechat, M.I. (2009) Estimated cumulative radiation dose from PET/CT in children with malignancies: A 5-year retrospective review. Pediatric Radiology, 40, 681-686. doi:10.1007/s00247-009-1434-z

[25] Karmazyn, B., Frush, D.P., Applegate, K.E., Maxfield, C., Cohen, M.D. and Jones, R.P. (2009) CT with a computer-simulated dose reduction technique for detection of pediatric nephroureterolithiasis: Comparison of standard and reduced radiation doses. American Journal of Roentgenology, 192, pp. 143-149. doi:10.2214/AJR.08.1391

[26] Hara, A.K., Paden, R.G., Silva, A.C., Kujak, J.L., Lawder, H.J. and Pavlicek, W. (2009) Iterative reconstruction technique for reducing body radiation dose at CT: Feasibility study. American Journal of Roentgenology, 193, 764-771. doi:10.2214/AJR.09.2397

[27] Hricak, H., Brenner, D.J., Adelstein, S.J., Frush, D.P., Hall, E.J., Howell, R.W., McCollough, C.H., Mettler, F.A., Pearce, M.S., Suleiman, O.H. et al. (2011) Managing radiation use in medical imaging: A multifaceted challenge. Radiology, 258, 889-905. doi:10.1148/radiol.10101157

[28] Lasio, G.M., Whiting, B.R. and Williamson, J.F. (2007) Statistical reconstruction for x-ray computed tomography using energy-integrating detectors. Physics in medicine and biology, 52, 2247-2266. doi:10.1088/0031-9155/52/8/014

[29] Linet, M.S., Kim, K.P. and Rajaraman, P. (2009) Children's exposure to diagnostic medical radiation and cancer risk: Epidemiologic and dosimetric considerations. $\mathrm{Pe}$ diatric Radiology, 39, 4-26. doi:10.1007/s00247-008-1026-3

[30] Tubiana, M. (2008) Computed tomography and radiation exposure. The New England Journal of Medicine, 358, 852-853.

[31] Huang, B., Law, M.W.-M. and Khong, P.-L. (2009) Wholebody PET/CT scanning: Estimation of radiation dose and cancer risk. Radiology, 251, 166-174. doi:10.1148/radiol.2511081300 